\section{Striate Cortex}

Islam Zaydan

Neurology, Virginia Commonwealth University, Richmond, VA, USA

\section{Synonyms}

Primary visual cortex; V1

\section{Definition}

The brain has more surface area dedicated to vision than any other sensory function. The primary visual receptive area is the striate cortex located in the occipital lobe. Also referred to as "V1," striate cortex is located in and around the calcarine fissure in each of the occipital lobes. V1 receives information directly from its ipsilateral lateral geniculate nucleus of the thalamus, via the optic radiations. It then sends information via two primary pathways: the "dorsal stream" and the "ventral stream." The dorsal stream, also known as the "where" or "how" pathway, projects to the parietal lobe and is thought to be involved in the location of objects in space. The ventral stream, also referred to as the "what" pathway, projects to the medial temporal lobe and limbic system, and is thought to be involved in object and form recognition.

\section{Cross-References}

- Calcarine Cortex

- Eye Fields

- Occipital Lobe

- Visual Cortex 